\title{
Light-diffracted intensities from very deep gratings
}

\author{
M. Nieto-Vesperinas and J. M. Soto-Crespo \\ Instituto de Optica, Consejo Superior de Investigaciones Científicas, Serrano 121, 28006-Madrid, Spain
}

(Received 16 March 1988)

\begin{abstract}
We show that very deep gratings may yield either enhanced specular or enhanced antispecular diffracted intensities when the direction of incidence coincides with that of an order of the diffraction pattern. Alternatively, for other angles of incidence the diffracted intensities are either strong in the specular or are uniformly distributed between all orders. Calculations are made for several profiles, all showing similar characteristics, in particular, an oscillatory behavior of the intensities with the amplitude of the corrugation.
\end{abstract}

\section{INTRODUCTION}

The phenomenon of enhanced backscattering of light has been predicted theoretically ${ }^{1,2}$ in connection with the effect of surface polariton localization in slightly rough random surface. One-dimensional numerical calculations for this case have also been reported. ${ }^{3,4}$

In addition, experimental observations of enhanced backscattered visible and infrared electromagnetic waves from very rough perfectly conductive random surfaces have recently been published. ${ }^{5,6}$ One-dimensional calculations to interpreting this effect were also made, ${ }^{7}$ concluding that in this case the enhancement results are due to pure interference between the elastically scattered waves.

These two aforementioned cases appear to be included in the general phenomenon of weak localization of photons in random media, also observed in light scattering from disordered volumes (a brief review with references may be found in Ref. 8).

As for the case of perfectly conductive surfaces, one may wonder how the interference effect will be affected by the geometry of the surface profile. In particular, one may ask what kind of diffraction pattern will be obtained if instead of a random surface one considers a grating, and whether a deep grating will produce enhanced antispecular orders. In fact, the existence of some blaze orders for some particular profiles of moderately rough gratings in the Littrow configuration has been known for sometime (see, e.g., Ref. 9 and references therein; see also Ref. 10). Also, it is known ${ }^{11}$ that for normal incidence the zeroth order tends to be dominant as the corrugation increases beyond a certain value since then the rainbow angles, ${ }^{12}$ at which intensity maxima occur, tend to move in towards the center of the diffraction diagram.

In this paper we show that when the direction of incidence of the wave coincides with that of a diffraction order (i.e., the order becomes antispecular), then the intensity of either this order or the specular may be very strongly enhanced for many appropriate combinations of the grating parameters. For other directions of incidence sometimes the specular is also very strong. (These facts do not seem to appear for random surfaces.) The enhancements can be more than $60 \%$ or even $80 \%$ of the total diffracted energy. The effect is observed for $2 h / a \gtrsim 0.4$ ( $h$ and $a$ being the amplitude and the period of the grating, respectively). This result shows that the blaze effect, so far obtained for a particular profile and particular order (usually the $n=-1$ ), is a much more general phenomenon when the grating is very deep; namely, we show that enhancement is obtained in all negative orders in Littrow mounting and, also for many profiles, as far as our computations reveal.

Calculations are made by means of an expansion of the
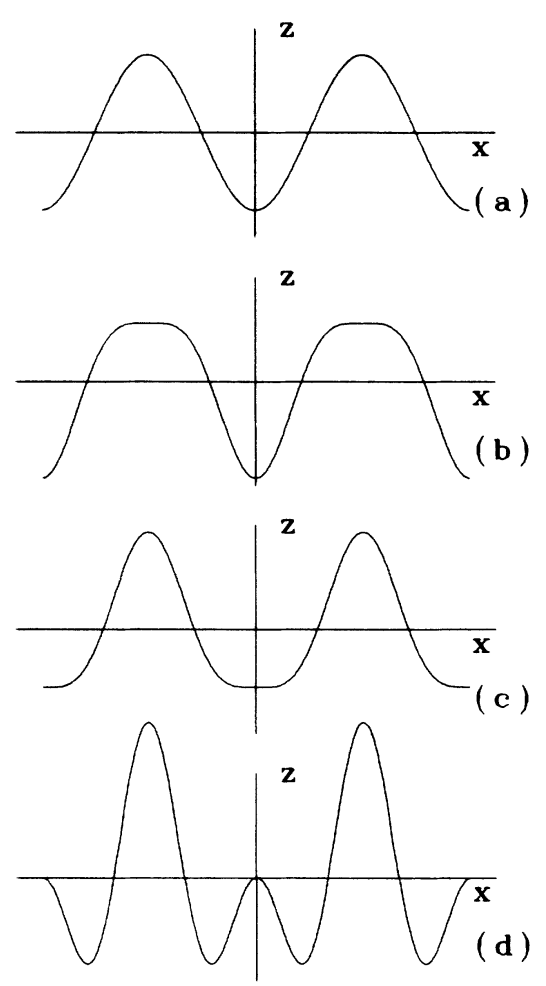

FIG. 1. Illustrating Eqs. (16): (a) $z=D_{1}(x)$, (b) $z=D_{2}(x)$, (c) $z=D_{3}(x)$, and (d) $z=D_{4}(x)$.

(c) 1988 The American Physical Society 

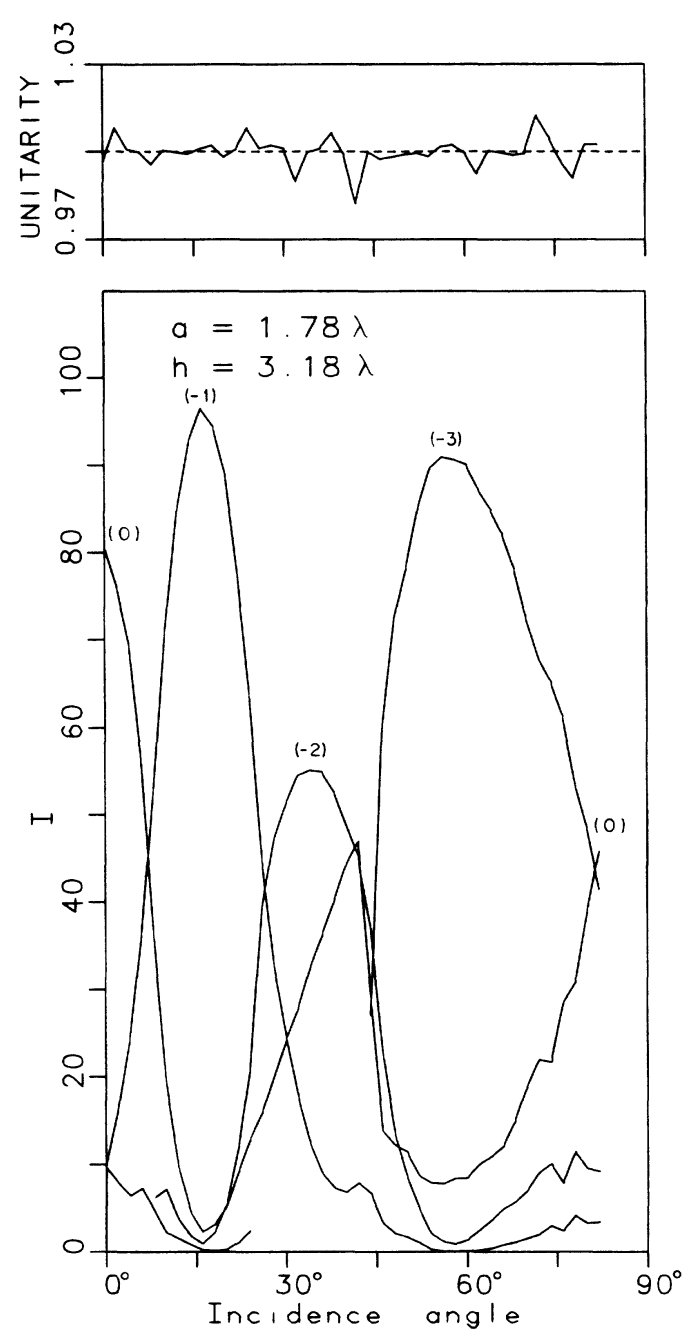

FIG. 2. Diffracted intensities vs angle of incidence $\theta_{0}$ for $D_{1}(x), a=1.78 \lambda, h=3.18 \lambda$.

Green function into Hankel functions, and by using the extinction theorem ${ }^{13}$ as a boundary condition. This procedure seems to be capable of dealing with corrugation amplitudes at least as high as those presented in Ref. 14.

In Sec. II we outline the method of calculation. $\mathrm{Nu}$ merical results for several profiles are shown in Sec. III.

\section{METHOD OF CALCULATION}

Let $z=D(x)$ be the surface grating profile, of period $a$, separating vacuum from a perfect conductor.

Let us consider an incident linearly polarized plane wave with wave vector

$$
\mathbf{k}_{0}=\left(K_{0}, 0, k_{0 z}\right) \text {, }
$$

with

$$
K_{0}^{2}+k_{0 z}^{2}=k_{0}^{2}, \quad k_{0}=\frac{2 \pi}{\lambda} .
$$

The electric and magnetic vectors are, respectively,
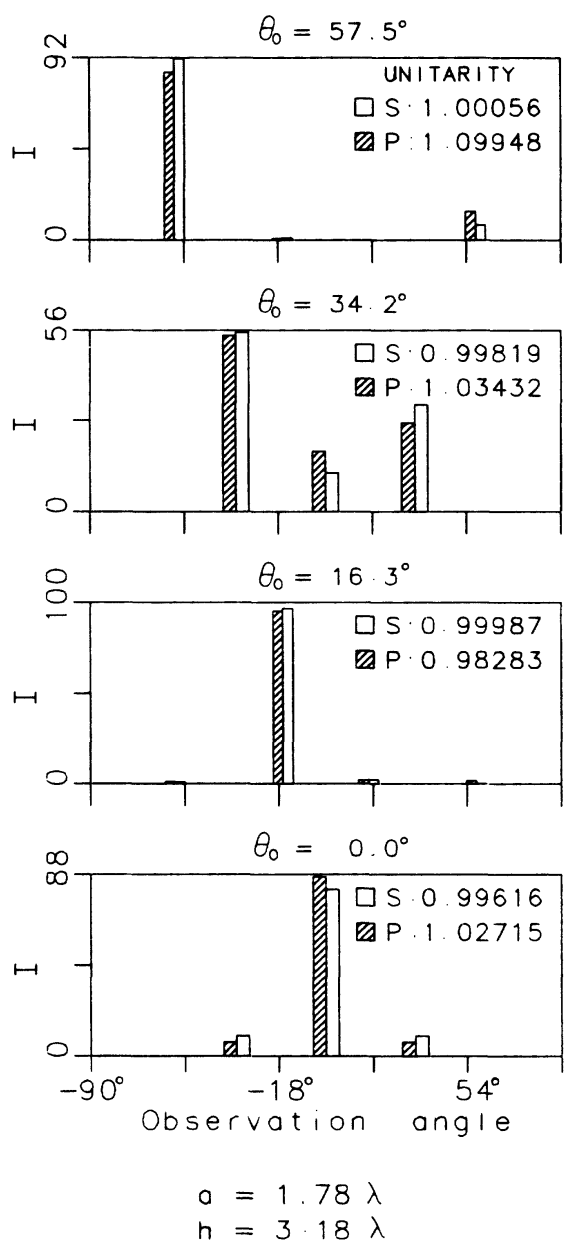

FIG. 3. Diffracted intensities at various angles of incidence $\theta_{0}$ for $D_{1}(x), a=1.78 \lambda, h=3.18 \lambda$.

given by

$$
\mathbf{E}^{(i)}=\mathrm{e} e^{i\left(K_{0} x-k_{0 z} z\right)}
$$

and

$$
\mathbf{H}^{(i)}=\mathbf{h} e^{i\left(K_{0} x-k_{0 z} z\right)}
$$

where the vectors $\mathbf{e}$ and $\mathbf{h}$ denote the directions of polarization. For $s$ waves ( $E$ perpendicular to the incidence plane), $\mathbf{e}$ is taken unitary. Reciprocally, $\mathbf{h}$ is considered unitary for $p$ waves ( $\mathbf{H}$ perpendicular to the incidence plane).

At points $\mathbf{r}_{<}=\left(x_{<}, 0, z_{<}\right)$below $z=D(x)$ the extinction theorem reads ${ }^{13}$ for $s$ waves and taking components (only along the $O y$ direction are different from zero)

$$
\begin{aligned}
E^{(i)}\left(\mathbf{r}_{<}\right)=\frac{\pi k_{0}}{c} \int & J_{y}\left(x^{\prime}\right) H_{0}^{(1)}\left(k_{0}\left|\mathbf{r}_{<}-\mathbf{r}^{\prime}\right|\right) \\
& \times\left[1+\left[\frac{\partial D}{\partial x^{\prime}}\right]^{2}\right]^{1 / 2} d x^{\prime},
\end{aligned}
$$

where $\mathbf{r}^{\prime}=\left(x^{\prime}, 0, D\left(x^{\prime}\right)\right) . J_{y}\left(x^{\prime}\right)$ is the electric current to 

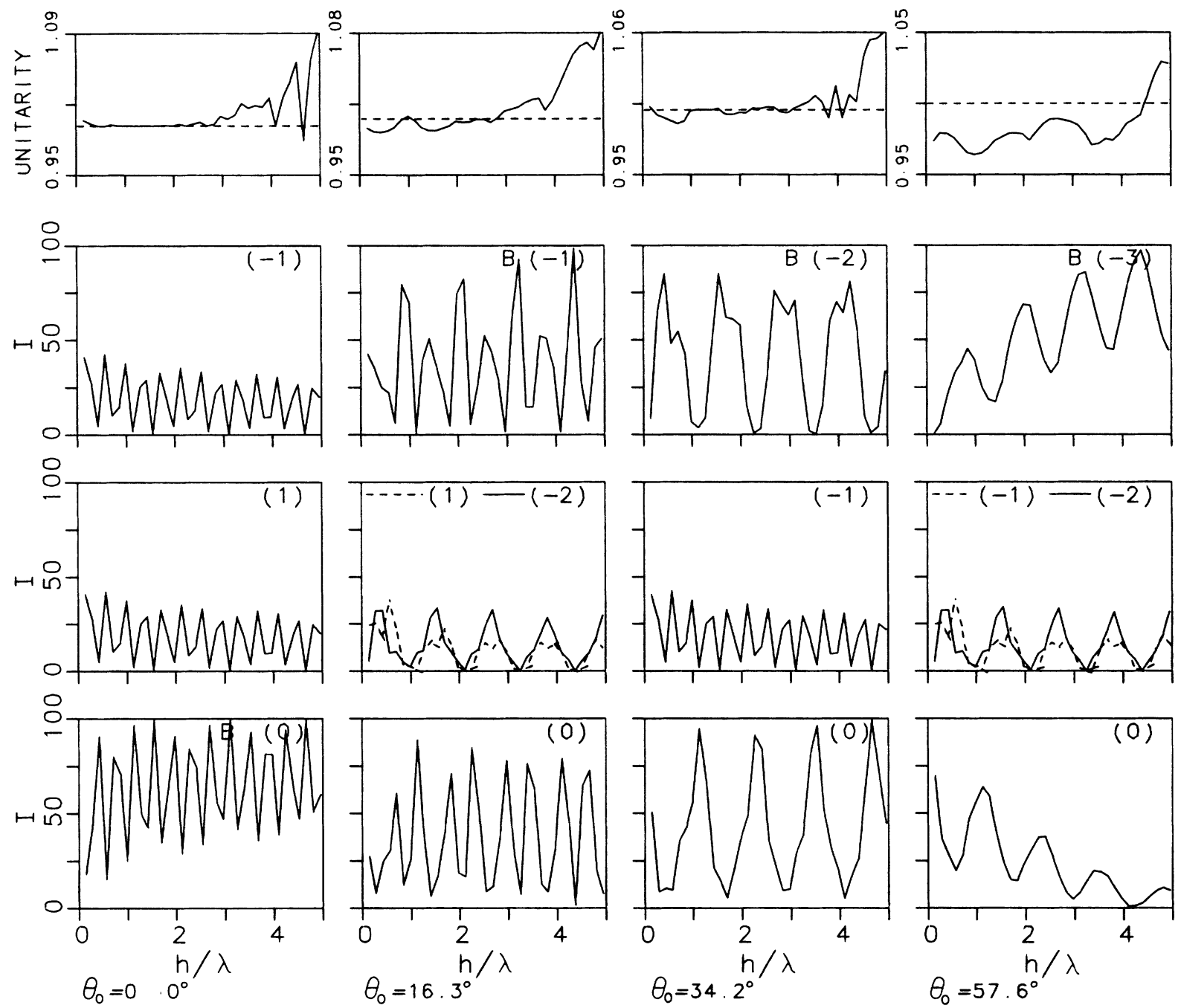

$a=1.78 \lambda$

(a)

FIG. 4. Diffracted intensities vs $h$ for $D_{1}(x), a=1.78 \lambda$, for (a) $\theta_{0}=0^{\circ}, 16.3^{\circ}, 34.2^{\circ}$, and $57.6^{\circ}$, at which an antispecular order exists (denoted $B$ ), and for (b) $\theta_{0}=9^{\circ}, 25^{\circ}$, and $46^{\circ}$, at which no antispecular order exists.

be obtained from the boundary condition [Eq. (4)] and $H_{0}^{(1)}$ is the zeroth-order Hankel function of the first kind.

By using the pseudoperiodicity of $J_{y}(x)$, namely

$$
J_{y}(x+a)=J_{y}(x) e^{i K_{0} a}
$$

Eq. (4) becomes

$$
\begin{array}{r}
E^{(i)}\left(\mathbf{r}_{<}\right)=\frac{\pi k_{0}}{c} \int_{0}^{a} J_{y}\left(x^{\prime}\right) G^{s}\left(k_{0}\left|\mathbf{r}_{<}-\mathbf{r}^{\prime}\right|\right) \\
\times\left[1+\left(\frac{\partial D}{\partial x^{\prime}}\right]^{2}\right]^{1 / 2} d x^{\prime} .
\end{array}
$$

Where the Green function for $s$ waves is given by the following expansion:

$$
G^{s}\left(k_{0}\left|\mathbf{r}_{<}-\mathbf{r}^{\prime}\right|\right)=\sum_{l=-\infty}^{\infty} H_{0}^{(1)}\left(k_{0}\left\{\left[x-\left(x^{\prime}+l a\right)\right]^{2}+\left[z-D\left(x^{\prime}\right)\right]^{2}\right\}^{1 / 2}\right) e^{i k_{0} l a} .
$$

Once $J_{y}\left(x^{\prime}\right)$ is found, the corresponding diffraction amplitudes are given by 

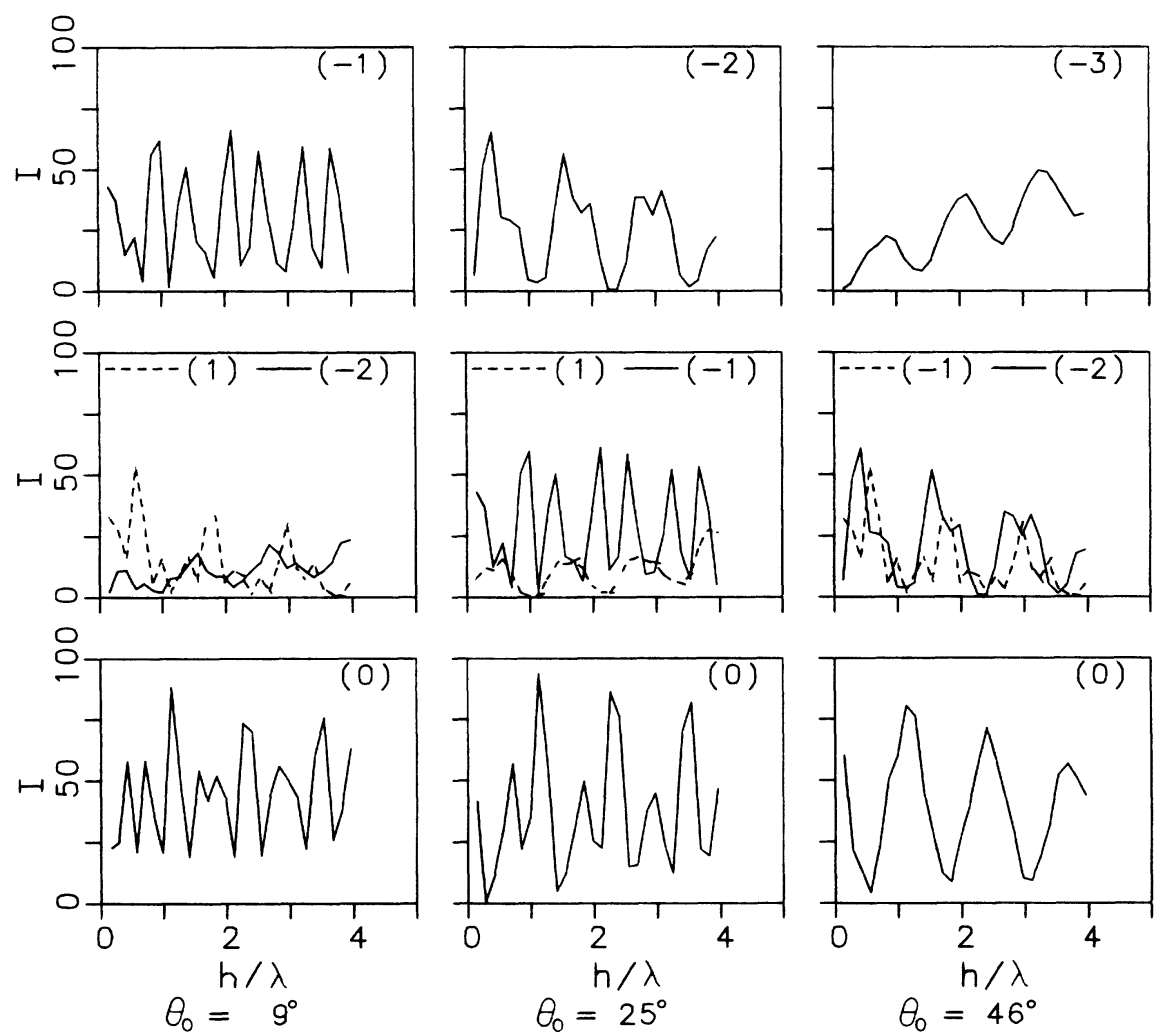

$a=1.78 \lambda$

(b)

FIG. 4. (Continued).

$$
A_{n}=\frac{2 \pi k_{0}}{c a k_{n z}} \int_{0}^{a} J_{y}\left(x^{\prime}\right) e^{-i\left[K_{n} x^{\prime}+k_{n z} D\left(x^{\prime}\right)\right]}\left[1+\left(\frac{\partial D}{\partial x^{\prime}}\right]^{2}\right]^{1 / 2} d x^{\prime}
$$

where

$$
K_{n}^{2}+k_{n z}^{2}=k_{0}^{2}
$$

and

$$
K_{n}=K_{0}+\frac{2 \pi}{a} n
$$

Analogously, for $p$ waves, the extinction theorem reads

$$
H^{(i)}\left(\mathbf{r}_{<}\right)=-\frac{\pi i k_{0}}{c} \int_{0}^{a} J_{x}\left(x^{\prime}\right) G^{P}\left(k_{0}\left|\mathbf{r}_{<}-\mathbf{r}^{\prime}\right|\right)\left[1+\left(\frac{\partial D}{\partial x^{\prime}}\right]^{2}\right]^{1 / 2} d x^{\prime}
$$

where the Green function for $p$ waves is given by the expansion

$$
G^{P}\left(k_{0}\left|\mathbf{r}_{<}-\mathbf{r}^{\prime}\right|\right)=\sum_{l=-\infty}^{\infty} H_{1}^{(1)}\left(k_{0}\left\{\left[x-\left(x^{\prime}+l a\right)\right]^{2}+\left[z-D\left(x^{\prime}\right)\right]^{2}\right\}^{1 / 2}\right) e^{i k_{0}^{l a}} \frac{\left[z-D\left(x^{\prime}\right)\right]-\frac{\partial D}{\partial x^{\prime}}\left[x-\left(x^{\prime}+l a\right)\right]}{\left\{\left[x-\left(x^{\prime}+l a\right)\right]^{2}+\left[z-D\left(x^{\prime}\right)\right]^{2}\right\}^{1 / 2}} .
$$

Then the corresponding diffracted amplitudes are

$$
B_{n}=-\frac{2 \pi}{c a k_{n z}} \int_{0}^{a} J_{x}\left(x^{\prime}\right) e^{-i\left[K_{n} x^{\prime}+k_{n z} D\left(x^{\prime}\right)\right]}\left[\frac{\partial D}{\partial x^{\prime}} K_{n}-k_{n z}\right]\left[1+\left(\frac{\partial D}{\partial x^{\prime}}\right]^{2}\right]^{1 / 2} d x^{\prime} .
$$



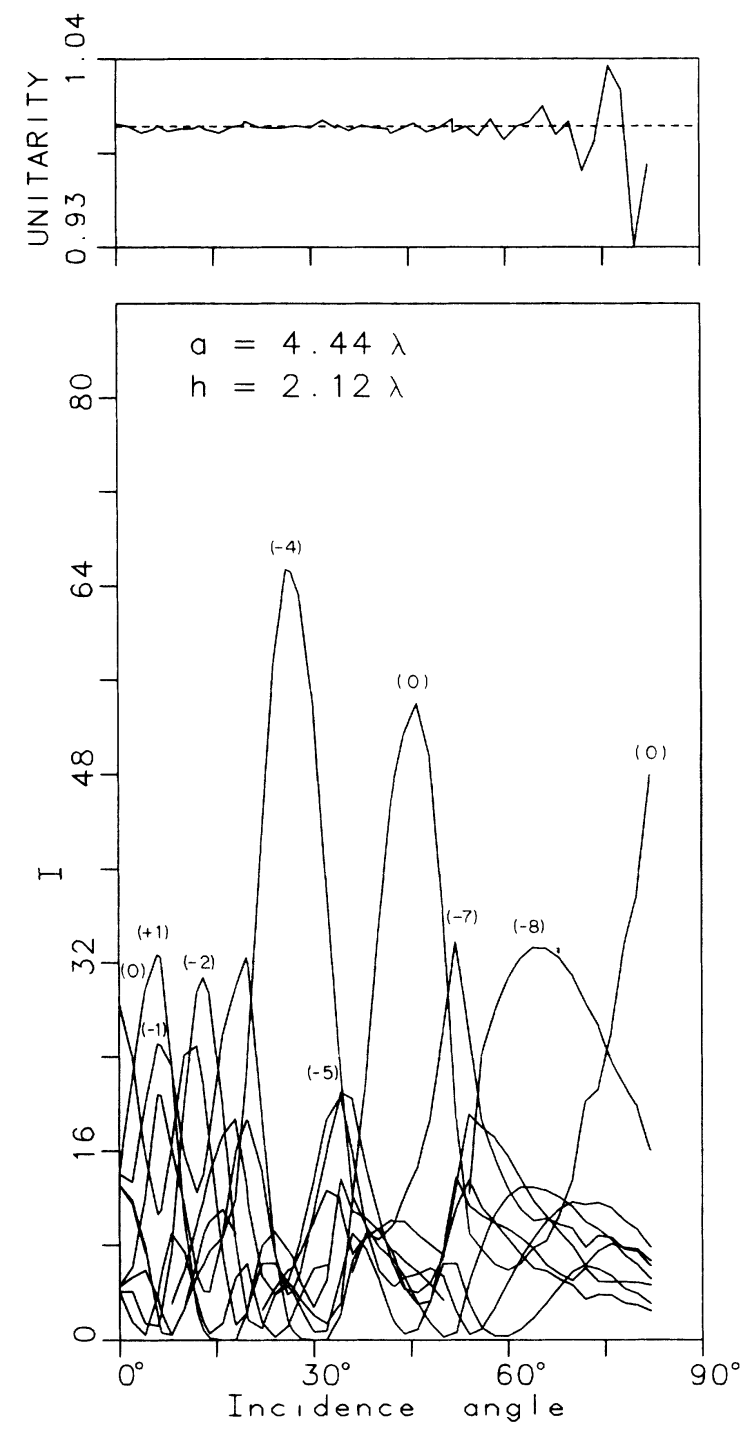

FIG. 5. Diffracted intensities vs angle of incidence $\theta_{0}$ for $D_{1}(x), a=4.44 \lambda, h=2.12 \lambda$.

The diffracted intensities, either for $s$ or $p$ waves (here $w=s$ or $p$ ), are given by

$$
I_{n}^{w}=\frac{k_{n z}}{k_{0 z}} \times \begin{cases}\left|A_{n}\right|^{2} & \text { for } s \text { waves } \\ \left|B_{n}\right|^{2} & \text { for } p \text { waves }\end{cases}
$$

and satisfy the unitarity condition:

$$
\sum_{n=-\infty}^{\infty} I_{n}^{w}=1
$$

Figure 1 shows the four different profiles used in the calculations:

$$
\begin{aligned}
& D_{1}(x)=h \cos \left(\frac{2 \pi}{a} x+\pi\right) \\
& D_{2}(x)=h \cos \left(\frac{2 \pi}{a} x+\pi\right)+\frac{h}{4} \cos \left(\frac{4 \pi}{a} x+\pi\right),
\end{aligned}
$$

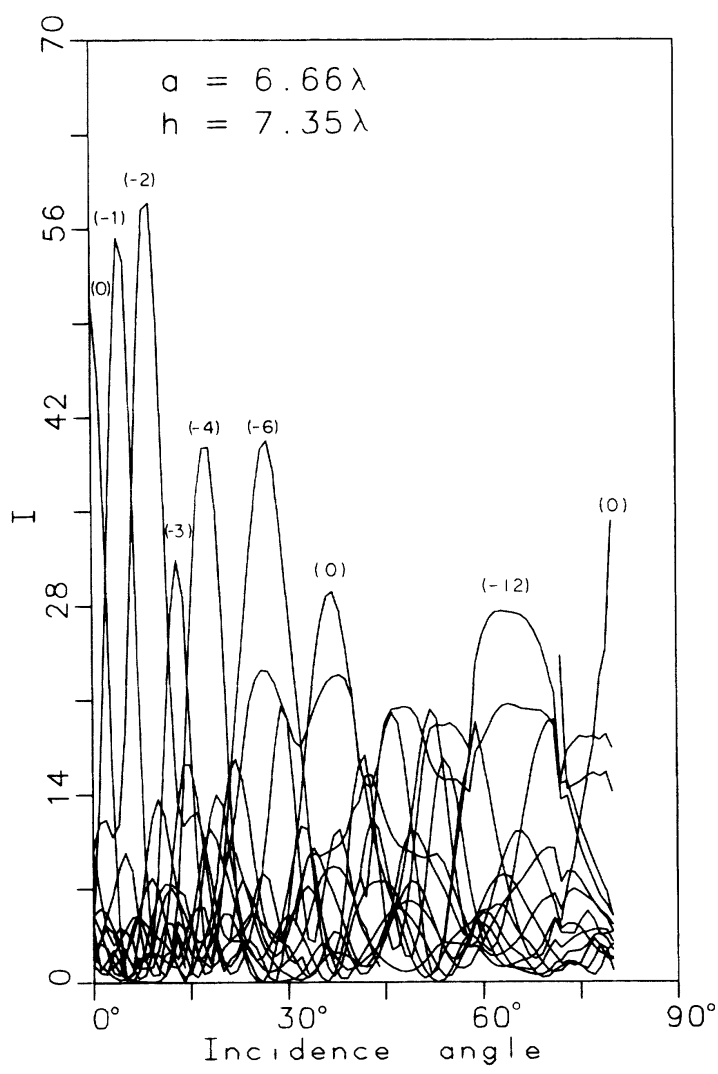

FIG. 6. Diffracted intensities vs angle of incidence $\theta_{0}$ for $D_{1}(x), a=6.66 \lambda, h=7.35 \lambda$.

$D_{3}(x)=\frac{h}{4} \cos \left(\frac{4 \pi}{a} x\right)-h \cos \left(\frac{2 \pi}{a} x\right)$,

$D_{4}(x)=h \cos \left(\frac{4 \pi}{a} x\right)-h \cos \left(\frac{2 \pi}{a} x\right)$.

\section{NUMERICAL RESULTS}

The series, Eqs. (7) or (12), are calculated by replacing $\sum_{l=-\infty}^{\infty}$ by $\sum_{l=-N}^{N}$. The number $N$ necessary to obtain convergence is controlled by criteria of unitarity and reciprocity. Typically, $N$ has values between $N=15$ and 40. The $x$ integration over one period is performed by sampling it into 220 points.

Figure 2 shows the diffracted intensities from the profile $D_{1}(x)$ versus the incidence angle $\theta_{0}$ for $s$ waves and for $h=3.18 \lambda$ and $a=1.78 \lambda$. Here an enhancement of the diffraction orders is seen when $\theta_{0}$ equals $0^{\circ}, 16.3^{\circ}$, $34.2^{\circ}$, and $57.6^{\circ}$. These are exactly the angles at which each of the corresponding orders becomes antispecular. The width of these peaks broadens as $n$ increases; this is due to the tendency of the diffracted energy to concentrate around backdiffraction directions when both $h$ and 

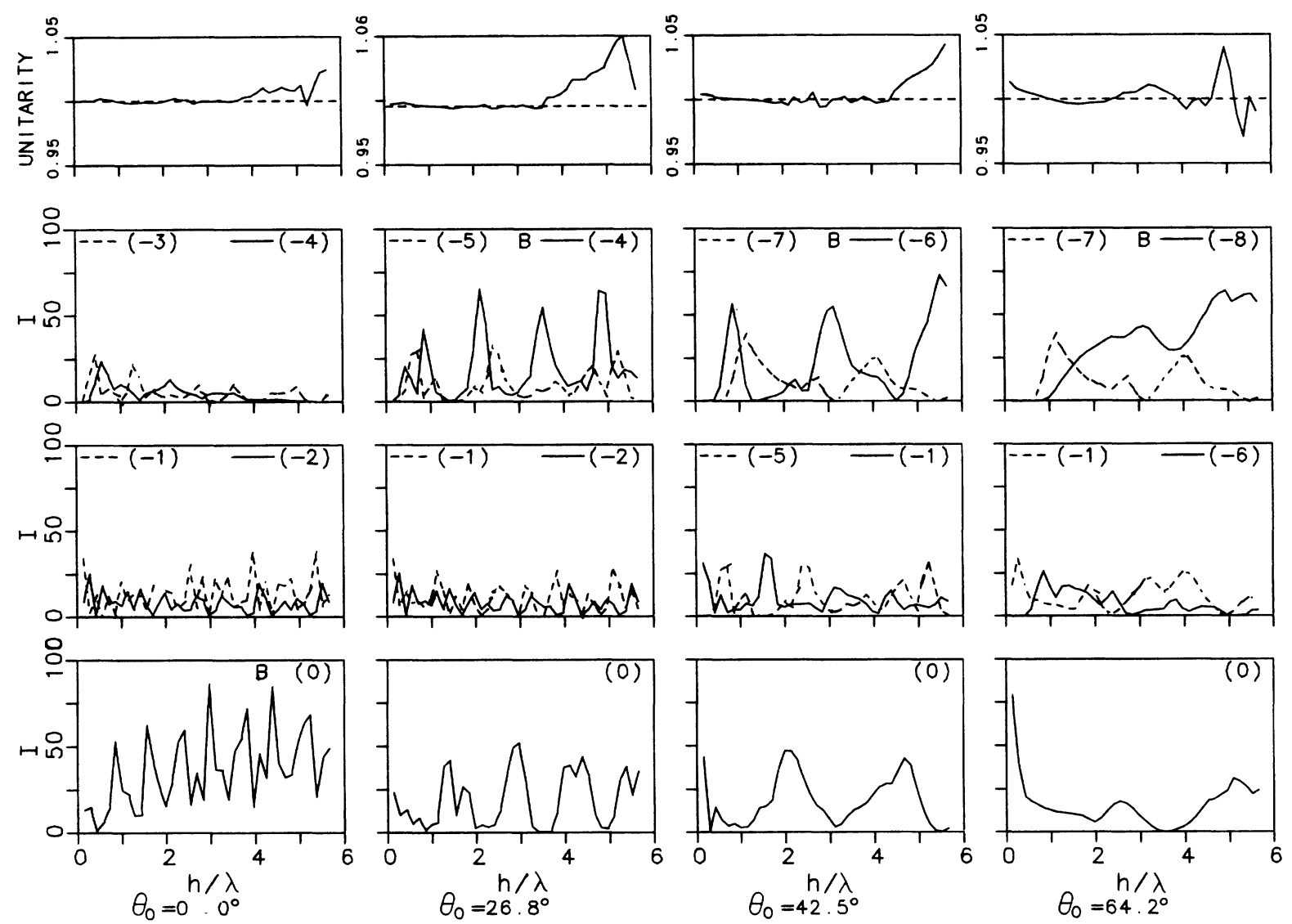

$a=4.44 \lambda$

FIG. 7. Diffracted intensities vs $h$ for $D_{1}(x), a=4.44 \lambda$, for $\theta_{0}=0^{\circ}, 26.8^{\circ}, 42.5^{\circ}$, and $64.2^{\circ}$, at which an antispecular order exists (denoted $B$ ).

$\theta_{0}$ are moderately large. Note that as $\theta_{0}$ approaches $90^{\circ}$ the specular tends to 1 as expected. For $h$ as large as in the cases considered here, there is not much difference between the diffraction patterns for $s$ waves and $p$ waves, in agreement with results obtained by other authors (see, e.g., Refs. 15 and 16). This is seen in Fig. 3 which shows the angular distribution of diffracted intensities. In particular, for the shallower surface $h=0.42 \lambda$ and $a=1.78 \lambda$, one obtains a $\theta_{0}$ diagram identical to that of Fig. 3(b) of Ref. 15. There only the $n=0$ and $n=-2$ intensities are appreciably enhanced.

One may gain insight by studying the dependence of the diffracted beams with $h$. Figure 4(a) shows this behavior for $\theta_{0}=0^{\circ}, 16.3^{\circ}, 34.2^{\circ}$, and $57.6^{\circ}$ ( $s$ waves). This oscillatory rainbow pattern of the orders (that was noted earlier ${ }^{17,18}$ for the zeroth and first dispersive orders) exhibits a large amount of the diffracted energy flowing forth and back between the specular and the antispecular. For incidence angles at which no antispecular exists [Fig. 4(b)], the specular also oscillates with high amplitudes, but at its minima the diffracted energy is shared more uniformly by all the other orders. As expected for a rainbow pattern, as $\theta_{0}$ increases, the period of the oscillations becomes larger. As seen, the unitarity is satisfactory up to $h \cong 3 \lambda$. Also, reciprocity holds, i.e., given the symme- try of the profile, the intensity $n=1 \quad\left(\theta=57.6^{\circ}\right)$ for $\theta_{0}=16.3^{\circ}$, for instance, equals that corresponding to $n=-1\left(\theta=16.3^{\circ}\right)$ for $\theta_{0}=57.6^{\circ}$. As another example, the intensities at $n=-2$ for $\theta_{0}=16.3^{\circ}$ and $\theta_{0}=57.6^{\circ}$ are equal.

As the grating period $a$ increases and more propagating orders appear, the peaks of the $\theta_{0}$ diagrams become sharper and more background of their tails is present. Figures 5 and 6 show the diffracted intensities versus $\theta_{0}$ for $s$ waves, for $a=4.44 \lambda$ and $h=2.12 \lambda$, and for $a=6.66 \lambda$ and $h=7.35 \lambda$, respectively. The $h$ dependence for the first of these two cases is shown in Fig. 7 for some intensities and for four incidence angles, $\theta_{0}=0^{\circ}, 26.8^{\circ}, 42.5^{\circ}$, and $64.2^{\circ}$, at which an antispecular order exists.

The effects quoted so far are not characteristics of sinusoidal gratings only. They also occur for other periodic profiles, as shown in what follows. For $z=D_{2}(x), a=1.78 \lambda$. Figure $8(\mathrm{a})$ depicts the $h$ dependence of the diffracted intensities for four angles $\theta_{0}$ at which an antispecular occurs. For other values of $\theta_{0}$ the results are shown in Fig. 8(b). For this particular profile, for which the apex is flatter than the bottom, the specular tends to dominate. This is more marked at larger $\theta_{0}$ where the specular is known to increase. For $z=D_{3}(x)$, 

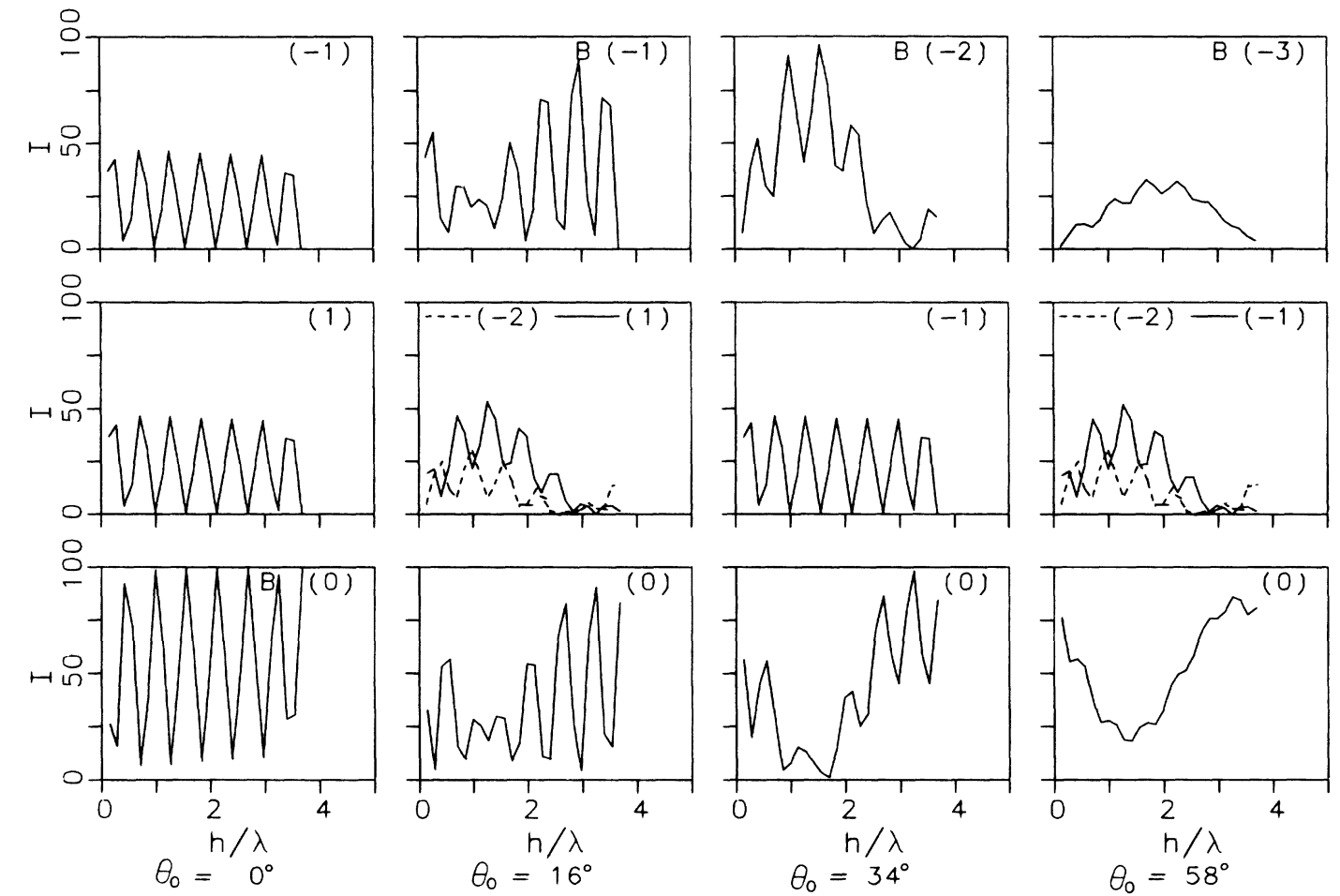

$a=1.78 \lambda$

(a)
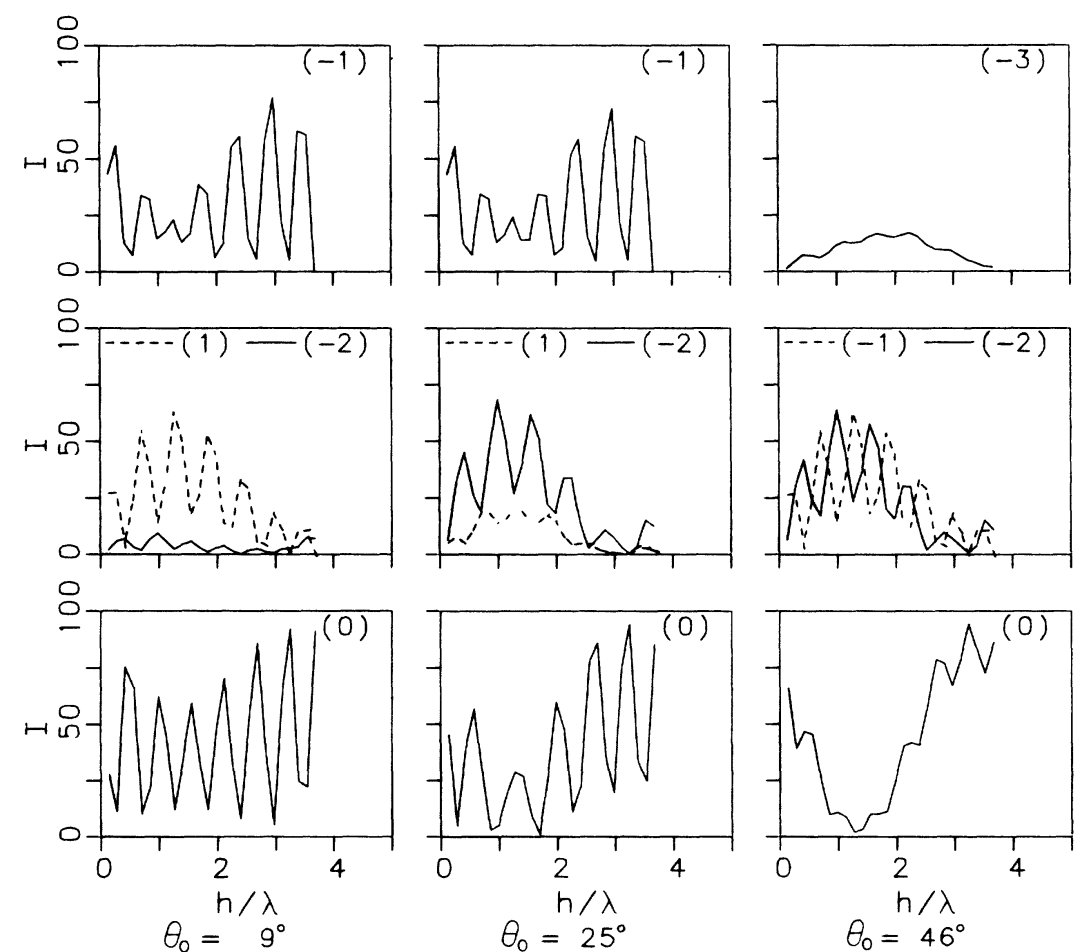

$a=1.78 \lambda$

(b)

FIG. 8. Diffracted intensites vs $h$ for $D_{2}(x), a=1.78 \lambda$, for (a) $\theta_{0}=0^{\circ}, 16.0^{\circ}, 34.0^{\circ}$, and $58.0^{\circ}$ (the antispecular order is denoted $B$ ) and for (b) $\theta_{0}=9^{\circ}, 25^{\circ}$, and $46^{\circ}$ (no antispecular order exists). 

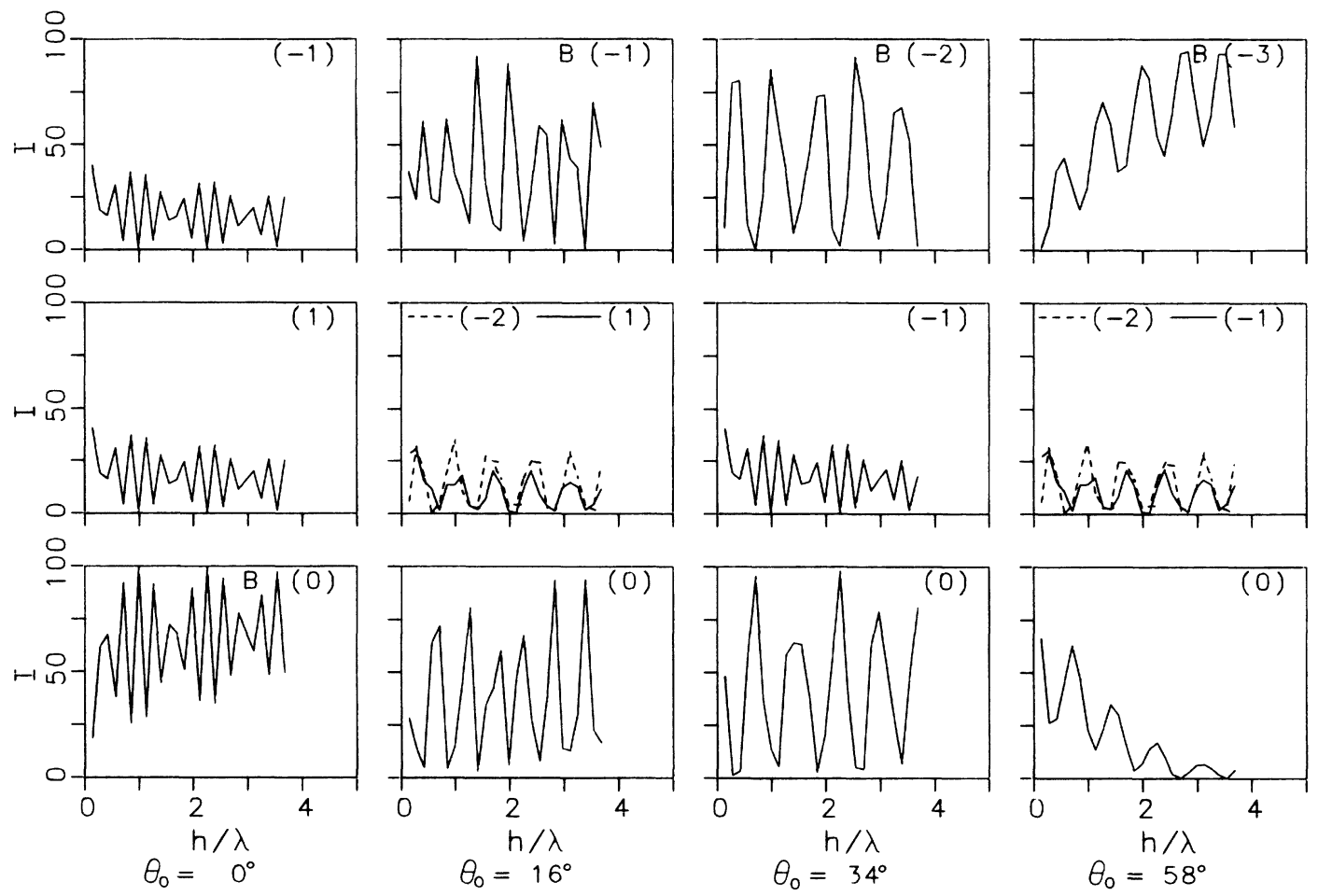

$a=1.78 \lambda$

(a)
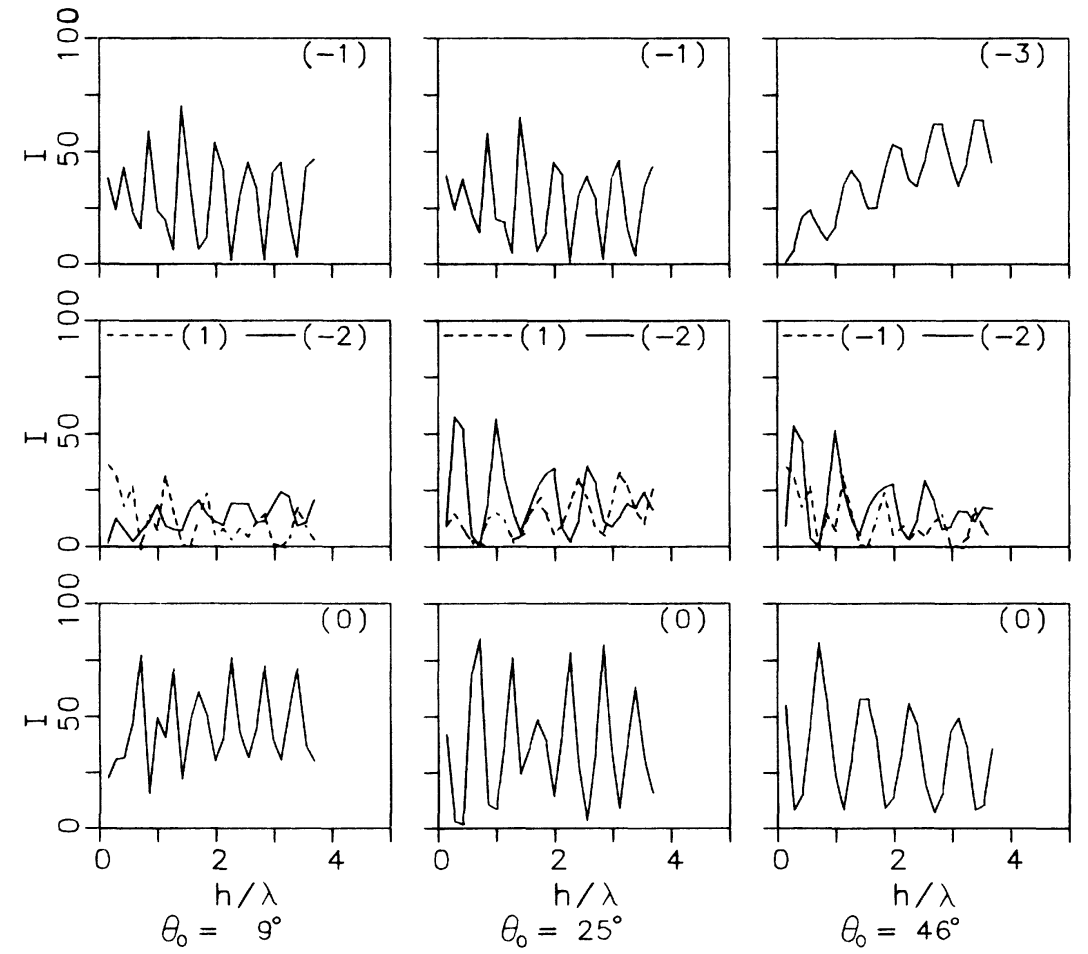

$a=1.78 \lambda$

(b)

FIG. 9. Diffracted intensities vs $h$ for $D_{3}(x), a=1.78 \lambda$, for (a) $\theta_{0}=0^{\circ}, 16.0^{\circ}, 34.0^{\circ}$, and $58.0^{\circ}$ (the antispecular order is denoted $B$ ) and for (b) $\theta_{0}=9^{\circ}, 25^{\circ}$, and $46^{\circ}$ (no antispecular order exists). 

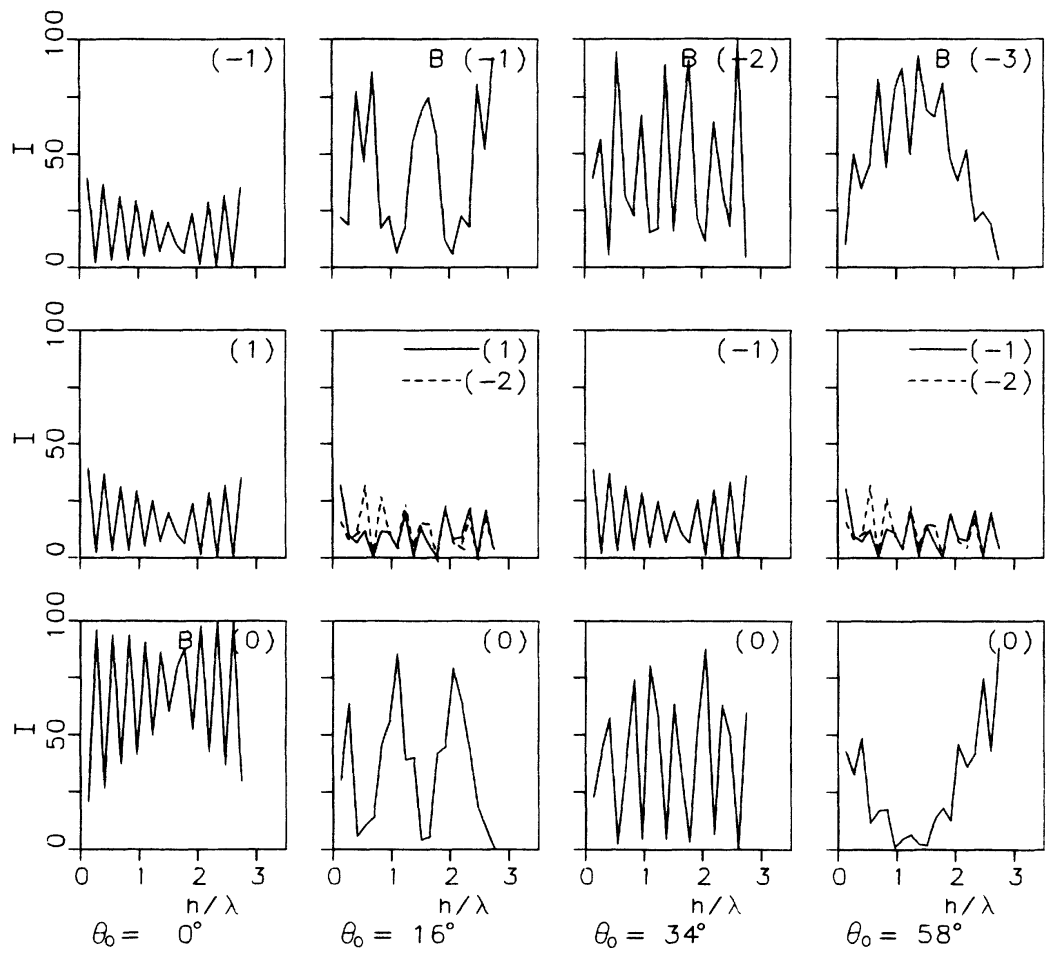

$a=1.78 \lambda$

(a)
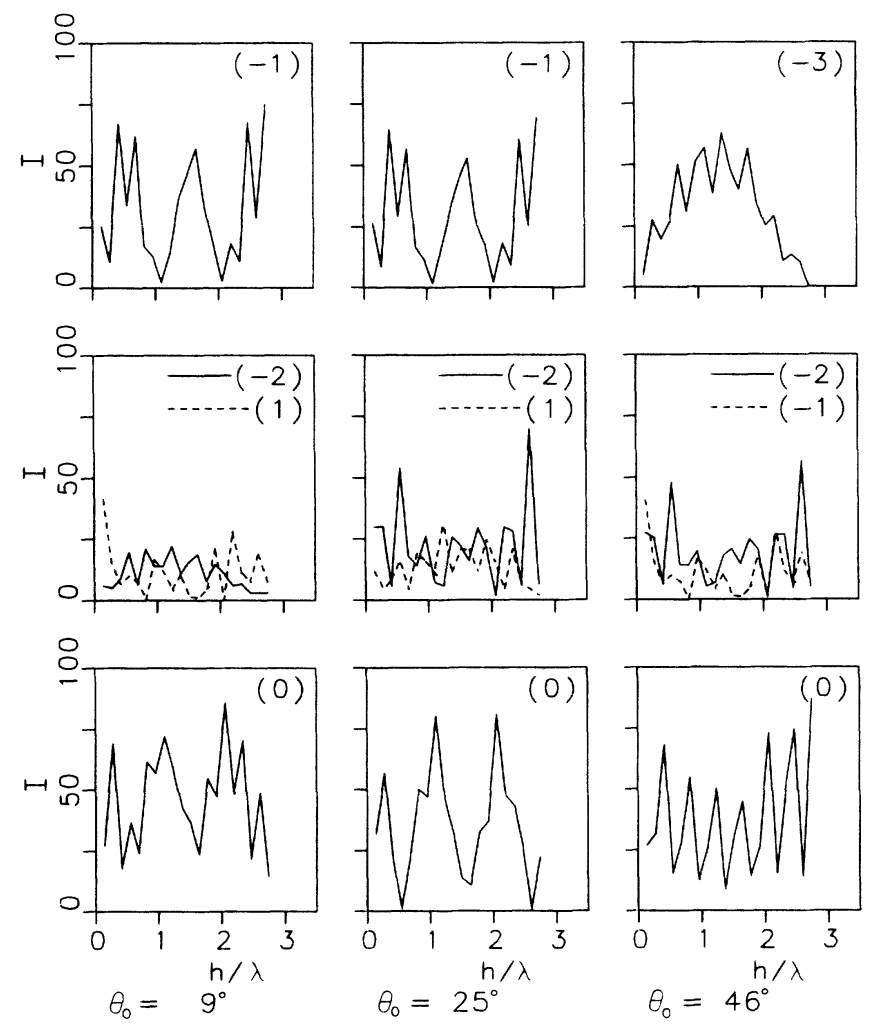

$a=1.78 \lambda$

(b)

FIG. 10. Diffracted intensities vs $h$ for $D_{4}(x), a=1.78 \lambda$, for (a) $\theta_{0}=0^{\circ}, 16.0^{\circ}, 34.0^{\circ}$, and $58.0^{\circ}$ (the antispecular order is denoted by $B$ ) and for (b) $\theta_{0}=9^{\circ}, 25^{\circ}$, and $46^{\circ}$ (no antispecular order exists). 
however, the antispecular is more enhanced, as shown by Fig. 9(a) $(a=1.78 \lambda)$. Once again, for incidence angles at which no antispecular intensity exists, the specular exhibits the same behavior as for the sinusoidal grating [Fig. 9(b)]. For other profiles the same effect remains. [See Fig. 10 for $z=D_{4}(x)$ and $a=1.78 \lambda$.]

\section{CONCLUSIONS}

In this paper we have dealt with very deep gratings. When the incidence direction coincides with that of an order, it has been shown that the grating may diffract very strongly, either in the antispecular or in the specular. For other incidence angles, the specular may also be very strong. The $h$ dependence shows a characteristic oscillatory behavior.
The connection with random surfaces might be understood by considering each random sample as one period of a supergrating. In this case, there are many propagating orders from this supergrating. Work is in progress to assess their distribution in the diffraction pattern, as well as to analyze the behavior of the specular and antispecular when the average over many samples is made.

\section{ACKNOWLEDGMENTS}

Useful discussions with N. García are greatly appreciated. This work has been supported by the Comision Interministerial de Ciencia y Tecnología, under Grant No. PB0278. J. M. S. acknowledges a grant from Ministerio de Educación y Ciencia.
${ }^{1}$ A. R. McGurn, A. A. Maradudin, and V. Celli, Phys. Rev. B 31, 4866 (1985).

${ }^{2}$ V. Celli, A. A. Maradudin, A. M. Marvin, and A. R. McGurn, J. Opt. Soc. Am. A 2, 2225 (1985).

${ }^{3}$ N. García and E. Stoll, J. Opt. Soc. Am. A 2, 2240 (1985).

${ }^{4}$ P. Tran and V. Celli (unpublished).

${ }^{5}$ E. R. Méndez and K. A. O'Donnell, Opt. Commun. 61, 91 (1987).

${ }^{6}$ K. A. O'Donnell and E. R. Méndez, J. Opt. Soc. Am. A 4, 1194 (1987).

${ }^{7}$ M. Nieto-Vesperinas and J. M. Soto-Crespo, Opt. Lett. 12, 979 (1987).

${ }^{8}$ S. J. Allen, Phys. Today 39(1), S-50 (1986).

${ }^{9}$ I. J. Wilson, L. C. Botten, and R. C. McPhedran, J. Opt. (Paris) 8, 217 (1977).

${ }^{10}$ D. Maystre, M. Neviere, and R. Petit, in Electromagnetic
Theory of Gratings, Vol. 22 of Topics in Current Physics, edited by R. Petit (Springer-Verlag, Berlin, 1980), Chap. 6.

${ }^{11}$ R. I. Masel, R. P. Merrill, and W. H. Miller, Phys. Rev. B 12, 5545 (1975).

12V. Garibaldi, A. C. Levi, R. Spadacini, and G. E. Tommei, Surf. Sci. 48, 649 (1975).

${ }^{13}$ D. N. Pattanayak and E. Wolf, Opt. Commun. 6, 217 (1972).

${ }^{14}$ N. García and N. Cabrera, Phys. Rev. B 18, 576 (1978).

${ }^{15}$ N. García and A. A. Maradudin, Opt. Commun. 45, 301 (1983).

${ }^{16}$ N. García, Opt. Commun. 45, 307 (1983).

${ }^{17}$ D. Maystre, M. Cadilhac, and J. Chandezon, Opt. Acta 28, 457 (1981).

${ }^{18}$ M. G. Moharam and T. K. Gaylord, J. Opt. Soc. Am. 72, 1385 (1982). 